\title{
Partial Multi-cell MMSE Vector Combining to Reduce Computational Cost for Massive MIMO Systems
}

\author{
Andrea P. Guevara, Cheng-Ming Chen, Sofie Pollin \\ Department of Electrical Engineering, KU Leuven, Belgium
}

\begin{abstract}
A practical partial multi-cell MMSE (PM-MMSE) combining vector for multi-cell massive MIMO systems is proposed in this work. This new scheme uses only inter-cell channels causing strong interference as partial inter-cell information in the multi-cell MMSE approach. The performance of PM-MMSE is evaluated using channels measured in a twocell outdoor experiment. In addition, a SIR threshold $\Gamma$ is introduced as a tradeoff parameter between spectral efficiency and computational cost. For multi-cell scenarios, simulation results show that in a system with 16 cells and 320 users, PM-MMSE is capable of achieving $96 \%$ of the M-MMSE spectral efficiency using on average a tenth of inter-cell channel information. For this scenario, PM-MMSE requires only $60 \%$ of the total number of multiplications used by M-MMSE.

Index Terms-Combining vector, computational cost, massive MIMO, spectral efficiency.
\end{abstract}

\section{INTRODUCTION}

Massive MIMO is one of the most promising new technologies and has shown to improve the spectral efficiency (SE) in both theoretical and real scenarios by serving multiple users at the same time and bandwidth [1]. The huge amount of antennas deployed in each base station (BS) boosts the spectral efficiency by overcoming the effects of fast fading and uncorrelated noise, and on top of that cancelling intra-cell interference with the use of linear precoders or combining vectors [2].

In a multi-cell scenario, during up-link (UL) transmission, a combining vector is used to coherently extract the desired signal from the received one. Different combining vectors, such as, maximum ratio combining (MRC), zero forcing (ZF) and regularized ZF (RZF) take into account only intracell information. In contrast, minimum-mean square error (MMSE) combining detectors consider not only intra-cell information but also the corresponding channel estimation errors and inter-cell information. When including inter-cell channels it exhibits the best performance.

Two different MMSE schemes, namely, single-cell MMSE (S-MMSE) and multi-cell MMSE (M-MMSE) detectors are proposed [3]. S-MMSE considers the interference caused by users within the analyzed cell (intra-cell interference), while M-MMSE includes also users from the other cells (intra-cell interference). The 'optimal' combining detector is M-MMSE, which maximizes the signal-to-interferencenoise ratio (SINR) by suppressing the inter-cell interference with instantaneous inter-cell channel information. For large systems, the SE gain of M-MMSE versus S-MMSE is up to $84 \%$ [3].

Nevertheless, the high computational cost that comes along with the M-MMSE combining vector challenges its implementation specially in large systems. In this work, we propose a new combining vector capable of reducing the computational cost without sacrificing SE. This scheme relies on channel selection based on a SIR heuristic to choose a subset of inter-cell channels with stronger interference to the desired cell. We name this method partial multi-cell MMSE (PMMMSE) due to the reduced amount of instantaneous inter-cell interference information required to determine the combining vector, and one can easily link this to the reduction in the computation cost. Moreover, the performance of PM-MMSE should be in between S-MMSE and M-MMSE depending on the accuracy in selecting the mentioned subset.

The rest of this paper is structured as follows: Section II describes the channel estimation process along with the SE per user and S-MMSE and M-MMSE combining vectors. Section III details the proposed PM-MMSE combing vector, specifically the inter-cell channel selection algorithm. The framework used to compute the computational cost is explained in Section IV. Section V describes a two-cell experiment setup and a multi-cell simulation system used to validate our proposed scheme. Numerical results are shown in Section VI and finally the main conclusions are summarized in Section VII.

The following notations are used in this paper: $\mathbf{x} \in \mathbb{C}^{\mathrm{M}}$ represents an $M \times 1$ complex vector, $\mathbf{X} \in \mathbb{C}^{\mathrm{M} \times \mathrm{N}}$ is an $M \times N$ complex matrix. $\mathbf{x}^{\mathrm{H}}$ and $\mathbf{x}^{\mathrm{T}}$ estates a transpose and conjugatetranspose of vector $\mathbf{x} .\|\mathbf{x}\|$ is the Euclidean norm of $\mathbf{x}$.

\section{Channel estimation AND SPECTRAL EFFiCIENCY}

In the following section a detail of our system model is presented, followed by a description of the two state of the art variations of the MMSE combining vector, S-MMSE and M-MMSE.

\section{A. System Model}

In this work we consider an UL time divison duplex (TDD) system composed of $L$ cells, each cell $j$ has a single BS with $M$ antennas and $K_{j}$ single antenna users (UEs). The wireless channel between UE $i$ in cell $l$ and BS in cell $j$ is $\mathbf{h}_{l i}^{j}$, with 
UE transmission power of $\mathrm{p}_{l i}$ and average channel gain per antenna given by:

$$
\beta_{l i}^{j}=10 \log _{10}\left\{\frac{\mathbb{E}\left\{\left\|\mathbf{h}_{l i}^{j}\right\|^{2}\right\}}{M_{j}}\right\} .
$$

Each UE channel is associated to an individual covariance matrix which contains statistic information about the spatial directions of their signal components and is obtained as follows:

$$
\mathbf{R}_{l i}^{j}=\mathbb{E}\left\{\mathbf{h}_{l i}^{j} \mathbf{h}_{l i}^{j \mathrm{H}}\right\} .
$$

\section{B. MMSE Channel Estimation}

During UL training, an orthogonal pilot sequence $\Phi_{j k}$ with length $\tau_{\mathrm{p}}{ }^{1}$ is allocated to each UE $k$ in cell $j$. Each cell $j$ also experiences pilot interference from $(L-1)$ adjacent cells plus noise $\mathbf{N} \sim \mathcal{N}\left(0, \sigma_{\mathrm{UL}}^{2}\right)$, therefore the signal at the receiver is:

$$
\mathbf{Y}_{j}=\sum_{k=1}^{K_{j}} \mathbf{h}_{j k}^{j} \Phi_{j k}^{\mathrm{T}}+\sum_{\substack{l=1 \\ l \neq j}}^{L} \sum_{i=1}^{K_{l}} \mathbf{h}_{l i}^{j} \Phi_{l i}^{\mathrm{T}}+\mathbf{N}
$$

Once the UL signal reaches a BS, it is correlated with the conjugate of each $j k$ pilot sequence for UE's channels that belong to the analyzed cell $j$, and the conjugate $l i$ pilot sequence for UEs of other cells.

$$
\mathbf{y}_{j k}^{j}=\mathbf{Y}_{j} \Phi_{j k}^{*} .
$$

If all the pilot sequences for all users in the $L$ cells are orthogonal, $\left(\Phi_{j k} \perp \Phi_{l i}\right)$ a non-pilot contamination scenario is considered and the received signal is decorrelated. In the case of pilot contamination the received signal will contain information with the desired and contaminated channels.

Once pilot decorrelation is carried out we can estimate each UE's channel. In this work the MMSE channel estimation based Theorem 3.1 from [4] is used as follows:

$$
\hat{\mathbf{h}}_{j k}^{j}=\sqrt{\mathrm{p}_{j k}} \mathbf{R}_{j k}^{j} \Psi_{j k} \mathbf{y}_{j k}^{j}
$$

where

$$
\Psi_{j k}=\left(\tau_{\mathrm{p}} \sum_{j^{\prime} k^{\prime} \in \xi_{j k}} \sqrt{\mathrm{p}_{j^{\prime} k^{\prime}}} \mathbf{R}_{j^{\prime} k^{\prime}}+\sigma_{\mathrm{UL}}^{2} \mathbf{I}_{M}\right)^{-1} .
$$

$\xi_{j k}$ represents the set of users in all $L$ cells that use the same pilot sequence. The noise variance $\sigma_{\mathrm{UL}}^{2}$ is obtained through the system signal-to-noise-ratio (SNR). And similar to (2), the covariance error matrix is:

$$
\mathbf{C}_{j k}^{j}=\mathbb{E}\left\{\left(\mathbf{h}_{j k}^{j}-\hat{\mathbf{h}}_{j k}^{j}\right)\left(\mathbf{h}_{j k}^{j}-\hat{\mathbf{h}}_{j k}^{j}\right)^{\mathrm{H}}\right\} .
$$

\footnotetext{
${ }^{1} \tau_{\mathrm{p}}$ UL data samples per coherence block are used to transmit a pilot sequence.
}

\section{MMSE Combining Vectors and Spectral Efficiency}

After UL pilot training, each BS receives an UL data signal which contains intra and inter-cell interference plus noise. To detect the desired signal for each UE $k$ in cell $j$ a combining vector $\mathbf{v}_{j k}$ is required. Therefore, for a cell $j$ its combining matrix is $\mathbf{V}_{j}=\left[\mathbf{v}_{j 1} \ldots \mathbf{v}_{j K}\right]$ and its estimated channel matrix $\hat{\mathbf{H}}_{j}^{j}=\left[\hat{\mathbf{h}}_{l 1}^{j} \ldots \hat{\mathbf{h}}_{l K_{l}}^{j}\right]$.

In this work, we analyze MMSE combining vector schemes as those are capable of maximizing the SINR and therefore increase SE [3]. On one hand, the M-MMSE combining vector uses the channel estimation information from all the users in the system, this scheme enhance the desired signal in the analyzed cell, and suppress all the intercell interference and noise.

$\mathbf{V}_{j}^{\mathrm{M}}=\left(\sum_{l=1}^{\mathrm{L}} \hat{\mathbf{H}}_{l}^{j} \mathbf{P}_{l}\left(\hat{\mathbf{H}}_{l}^{j}\right)^{\mathrm{H}}+\sum_{l=1}^{L} \sum_{i=1}^{K_{l}} p_{l i} \mathbf{C}_{l i}^{j}+\sigma_{\mathrm{UL}}^{2} \mathbf{I}_{M_{j}}\right)^{-1} \hat{\mathbf{H}}_{j}^{j} \mathbf{P}_{j}$

On the other hand, the S-MMSE scheme considers only its intra-cell channel information, treating the inter-cell information as uncorrelated noise.

$$
\mathbf{V}_{j}^{\mathrm{S}}=\left(\hat{\mathbf{H}}_{j}^{j} \mathbf{P}_{j}\left(\hat{\mathbf{H}}_{j}^{j}\right)^{\mathrm{H}}+\sum_{k=1}^{K_{j}} p_{j k} \mathbf{C}_{j k}^{j}+\sigma_{\mathrm{UL}}^{2} \mathbf{I}_{M_{j}}\right)^{-1} \hat{\mathbf{H}}_{j}^{j} \mathbf{P}_{j} .
$$

The performance of each combining vector scheme is analyzed as the achievable UL SINR and SE for each UE. Theorem 4.1 in [4] propose an instantaneous SINR which considers channel estimation error in (10) and its SE in (11):

$$
\operatorname{SINR}_{j k}^{\mathrm{UL}}=\frac{p_{j k}\left|\mathbf{v}_{j k}^{\mathrm{H}} \hat{\mathbf{h}}_{j k}^{j}\right|^{2}}{\sum_{\substack { l=1 \\
\begin{subarray}{c}{i=1 \\
l \neq j \\
i \neq k{ l = 1 \\
\begin{subarray} { c } { i = 1 \\
l \neq j \\
i \neq k } }\end{subarray}}^{K_{l}} p_{l i}\left|\mathbf{v}_{j k}^{\mathrm{H}} \hat{\mathbf{h}}_{l i}^{j}\right|^{2}+\mathbf{v}_{j k}^{\mathrm{H}}\left(\sum_{l=1}^{L} \sum_{i=1}^{K_{l}} p_{l i} \mathbf{C}_{l i}^{j}+\sigma_{\mathrm{UL}}^{2} \mathbf{I}_{M_{j}}\right) \mathbf{v}_{j k}}
$$

$$
\mathrm{SE}_{j k}=\frac{\tau_{u}}{\tau_{c}} \mathbb{E}\left\{\log _{2}\left(1+\operatorname{SINR}_{j k}^{\mathrm{UL}}\right)\right\} .
$$

Where $\tau_{\mathrm{u}} / \tau_{\mathrm{c}}{ }^{2}$ is the portion per coherence block used to transmit UL data.

\section{PM-MMSE VECTOR COMBINING}

From the previous combining vectors it is noticed that the inter-cell information is treated as all or nothing. Despite the fact that M-MMSE considers all inter-cell channels, not all of them contribute to achieve a high spectral efficiency. The proposed PM-MMSE scheme selects only those intercell channel with strong interference.

\footnotetext{
${ }^{2} \tau_{\mathrm{u}}$ is the UL data samples received per coherence block, and $\tau_{\mathrm{c}}$ is the total number of samples per coherence block
} 


\section{A. Heuristic Multi-cell Interference}

After channel estimation, PM-MMSE algorithm selects all inter-cell UE $i$ in all cells $l$ whose heuristic $\operatorname{SIR}_{l i}^{j}$ relative to the analyzed cell $j$ is below a SIR system threshold $\Gamma$ :

$$
\operatorname{SIR}_{l i}^{j}=\max \left(\hat{\beta}_{j k}^{j}\right)-\hat{\beta}_{l i}^{j} \leq \Gamma \quad(\mathrm{dB}) \quad l \neq j .
$$

To obtain the $\operatorname{SIR}_{l i}$ in cell $j$, we first select the UE $k$ in that cell $j$ with the best channel gain to the BS in cell $j$ as a reference signal mainly to normalize the power levels and have a reference point. Note that we could also have chosen the mean power or minimum power in a cell as reference point. We use this reference signal $\left(\hat{\beta}_{j k}^{j}\right)$ then to compare the relative power of all other UE in the system using (12). This relative $\mathrm{SIR}_{l i}$ is used as a heuristic to determine if the UE $i$ in cell $l$ should be considered as an inter-cell interferer in the PM-MMSE scheme. A UE is an interferer if it would cause the SIR heuristic to be below a certain threshold.

Along with the selected channels their covariance error matrix and transmission power are also included for further processing in PM-MMSE as it is described in Algorithm 1.

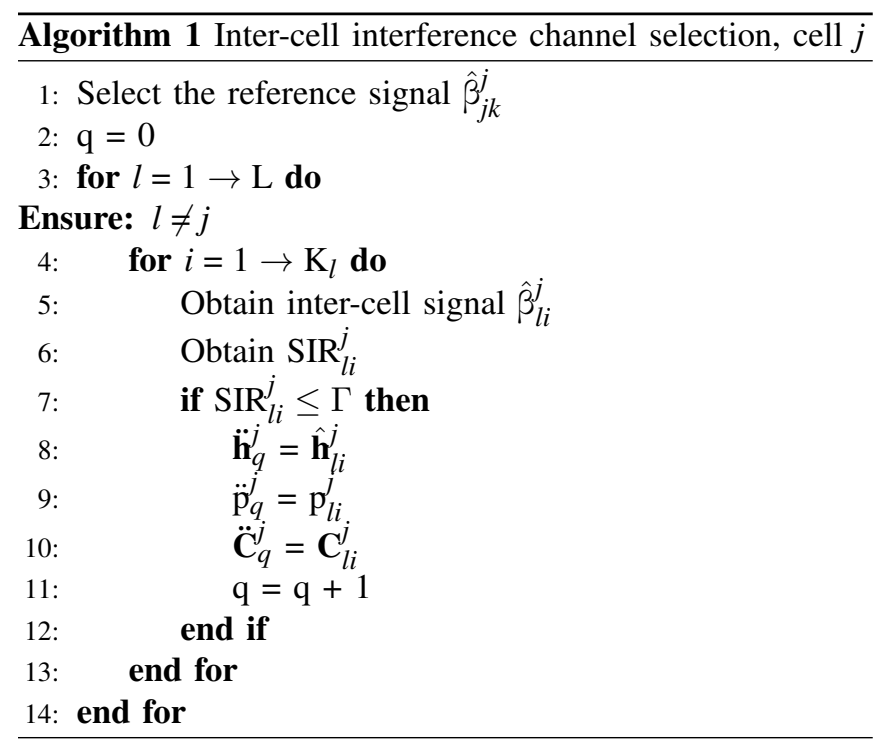

The total number of selected channels for cell $j$ is $Q^{j}$. Then, the partial multi-cell channel matrix $\ddot{\mathbf{H}}^{j}$ and partial multi-cell power matrix $\ddot{\mathbf{P}}^{j}$ for cell $j$ are presented in (13) and (14).

$$
\begin{gathered}
\ddot{\mathbf{H}}^{j}=\left[\ddot{\mathbf{h}}_{1}^{j}, \ddot{\mathbf{h}}_{2}^{j}, \ldots, \ddot{\mathbf{h}}_{Q}^{j}\right]^{\mathrm{T}} . \\
\ddot{\mathbf{p}}^{j}=\operatorname{diag}\left(\ddot{\mathrm{p}}_{1}^{j}, \ddot{\mathrm{p}}_{2}^{j}, \ldots, \ddot{\mathrm{p}}_{Q}^{j}\right) .
\end{gathered}
$$

\section{B. Importance of SIR Threshold $\Gamma$}

$\Gamma$ is a key parameter for inter-cell interference selection and determines how large the (estimated) interference should be before considering it in PM-MMSE. The SIR threshold could be used as a tradeoff metric between SE and computational cost. So, when $\Gamma \rightarrow-\infty$ no inter-cell channels are considered in PM-MMSE, then $Q \rightarrow 0$ and PM-MMSE $\rightarrow$ SMMSE. On the other hand, when $\Gamma \rightarrow+\infty$ all inter-cell channels are considering for PM-MMSE, then $Q \rightarrow(L-1) K$ and PM-MMSE $\rightarrow$ M-MMSE.

\section{Partial Multi-cell MMSE combining vector (PM-MMSE)}

We categorize the intra and inter-cell interference accounted for PM-MMSE in (15) similar to M-MMSE in (8). The first term is the regular intra-cell interference. Notwithstanding, inter-cell information will be selected according to Algorithm 1. So, in PM-MMSE only $Q$ inter-cell channels and their covariance error matrices are considered as intercell information, second term in (15).

\section{Computational cost}

The computational cost is quantified as the number of complex multiplications and divisions ${ }^{3}$ required per coherence block to compute a desired signal vector. In [4] Lemma B.1 and B.2 describe the number of complex multiplications by each operation used in a MMSE combining vector scheme.

First, $\left(M^{2}+M\right) K / 2$ number of multiplications are required to compute a channel matrix with its transpose conjugate, $\mathbf{H H}^{\mathrm{H}}$. In Table I we can see that M-MMSE repeats this process $L$ times for all channels in the system, while PMMMSE add to the analysed cell only $Q$ inter-cell channels. Second, the inverse term of any MMSE combining vector requires $\left(M^{3}+M\right) / 3$ complex multiplications. Finally, the cost between the mentioned inverse term and HP is $M^{2}$ times the total number of channels considering per combining vector.

Thus, the total number of complex multiplications per coherence block includes $\tau_{\mathrm{u}} M K$ plus the following complex multiplications ${ }^{4}$.

TABLE I

COMPUTATIONAL COMPLEXITY REQUIRED PER COMBINING VECTOR.

\begin{tabular}{|c|c|}
\hline M-MMSE & $\left(3 M^{2}+M\right) L \frac{K}{2}+\left(M^{3}-M\right) \frac{1}{3}$ \\
\hline S-MMSE & $\left(3 M^{2}+M\right) \frac{K}{2}+\left(M^{3}-M\right) \frac{1}{3}$ \\
\hline PM-MMSE & $\left(3 M^{2}+M\right) \frac{K+Q}{2}+\left(M^{3}-M\right) \frac{1}{3}+K(L-1)$ \\
\hline
\end{tabular}

Assuming that all channel gains are already known by the system, the operations needed to carry out channel selection in Section III-A contribute only $K(L-1)$ additional complex multiplications when (12) is compute linearly.

\section{Simulation AND EXPERIMENT SETUP}

To analyse the performance of the proposed PM-MMSE combining vector in a two-cell scenario using realistic channel and interference estimates, we carried out an outdoor experiment deployed in the KULeuven massive MIMO testbed.

\footnotetext{
${ }^{3}$ Additions and subtractions also contribute to the computational cost, however, their contribution is small and are neglected in this work.

${ }^{4}$ Considering a homogeneous system, with $M$ number of antennas for all BS and $K$ users for all cells.
} 


$$
\mathbf{V}_{j}^{\mathrm{P}}=(\underbrace{\hat{\mathbf{H}}_{j}^{j} \mathbf{P}_{j}\left(\hat{\mathbf{H}}_{j}^{j}\right)^{\mathrm{H}}+\sum_{i=1}^{K_{j}} p_{j i} \mathbf{C}_{j i}^{j}}_{\text {Intra-cell information }}+\underbrace{\ddot{\mathbf{H}}^{j} \ddot{\mathbf{P}}^{j}\left(\ddot{\mathbf{H}}^{j}\right)^{\mathrm{H}}+\sum_{q=1}^{Q^{j}} \ddot{p}_{q}^{j} \ddot{\mathbf{C}}_{q}^{j}}_{\text {Partial Inter-cell information }}+\sigma_{\mathrm{UL}}^{2} \mathbf{I}_{M_{j}})^{-1} \hat{\mathbf{H}}_{j}^{j} \mathbf{P}_{j}
$$

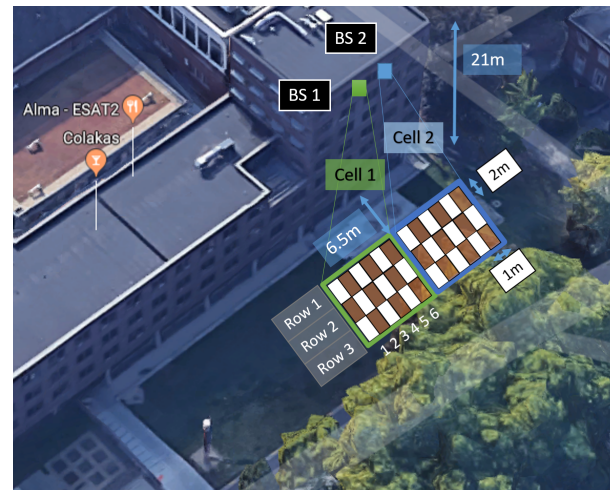

Fig. 1. Overlook of the symmetric two-cell Massive MIMO outdoor scenario at ESAT KU Leuven. The location of each BS is 21 meters heigh, separated by 6.5 meters. Each cell supports $18 \mathrm{UEs}$, which are distributed in 3 rows ( 2 meters) and 6 columns (1 meter).

While for multi-cell scenarios we used line-of-sight (LOS) massive MIMO simulations.

\section{A. Experiment Setup}

The distributed KULeuven massive MIMO testbed used in an outdoor two-cell experiment is described in detail in [5]. This testbed is supported by National Instrument universal software radio peripherals (USRPs) and controlled in real time by the LabVIEW Communications MIMO Application Framework 1.1. [6], operating at $2.61 \mathrm{GHz}$ with LTE-TDD standard. The two main components of this system are the distributed BS and fixed UEs.

As a unique feature of this testbed, the 64-antennas from the BS are distributed into two separate patch antenna arrays of equal size. For this experiment, both arrays were placed at the rear windows of KULeuven-ESAT building on the fifth floor at 21 meters high, separated with 6.5 meters from each other and titled $24^{\circ}$.

The second part of this setup comprises a set of fixed UEs. Those UEs are implemented in a dual-antenna USRP that is capable to transmit two data streams simultaneously. Each stream and antenna are associated to a single UE. In this experiment, we used dipole antennas for each UE, with a TX power of $20 \mathrm{dBm}$. Attached to each dipole antenna was a power amplifier to boost its power for an extra $13 \mathrm{~dB}$.

One USRP was placed symmetrically in one of the 18 grid locations on the ground floor at the rear side of KULeuvenESAT building, facing both BS arrays. This experiment collected the uplink UE's channel in every location and after channel processing we associate 18 users to one BS array, creating a two cells system, as it is shown in Fig. 1. Therefore this system has two homogeneous cells $(L=2)$ with $K=18$

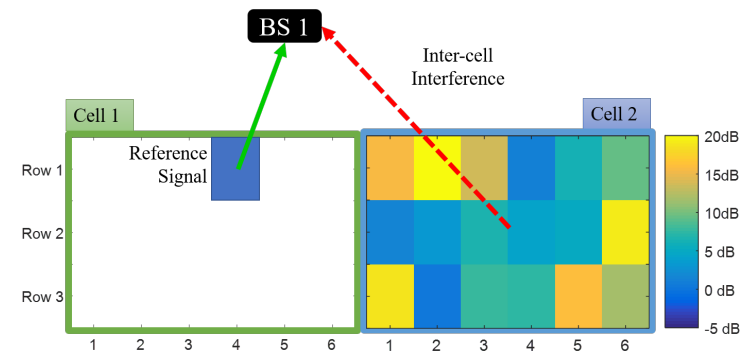

Fig. 2. Inter-cell SIR for each UE from cell 2 to BS 1 . Where the UE in cell 1 row 1 column 4 has the strongest channel gain and is consider as the reference signal based on (12). Notice that a UE in cell 2 giving rise to a SIR of $20 \mathrm{~dB}$ for the reference user, means that the interference generated by that UE in cell 2 is $20 \mathrm{~dB}$ larger than the reference signal.

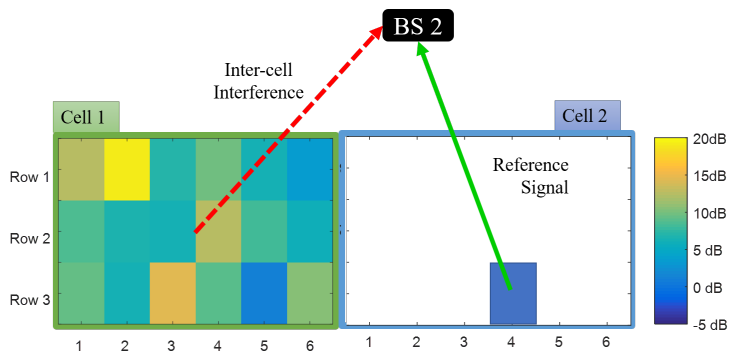

Fig. 3. Inter-cell SIR for each UE from cell 1 to BS 2. Where the UE in cell 2 row 3 column 4 has the strongest channel gain and is considered as the reference signal for SIR in cell 1 based on (12).

and $M=32$ for each cell. All inter- and intra-cell channels were measured, so we can evaluate inter-cell interference and the performance of M-MMSE using the measured channel database.

1) Channel collection: During our experiment, the channel of each UE was collected for 1 OFDMA symbol of 100 sub-carriers every $100 \mathrm{~ms}$, during 20 seconds. This channel was treated as raw channel for the following data processing, albeit it was not normalized in any kind, to preserve its path gain.

2) Data processing: As a first step during data processing, a fixed noise power was added to all UE, setting each UE's SNR between $30 \mathrm{~dB}$ to $40 \mathrm{~dB}$, depending solely on their channel gain $\beta_{j k}$ as the UL power $p$ is the same for all of them.

$$
\mathrm{SNR}_{j k}=\frac{p \beta_{j k}}{\sigma^{2}}
$$

Once SNR was set, channel estimation process was carried out, continuing with the computation of different MMSE combining vectors and later with SE. 


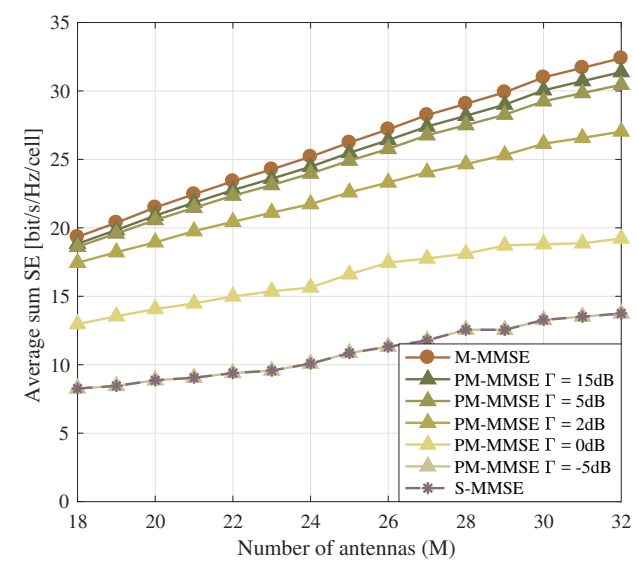

Fig. 4. Average UL SE comparison for different values of $\Gamma$ for PM-MMSE combining vector, as a function of the number of antennas per BS, applied to our experiment

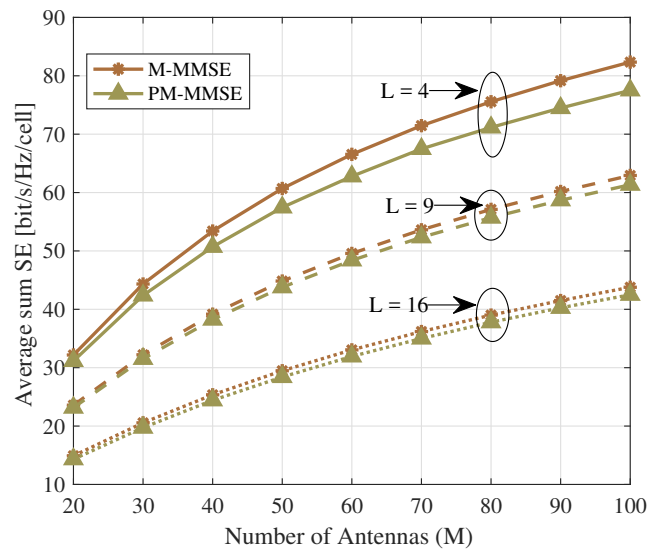

Fig. 5. Average UL SE per cell, for M-MMSE and PM-MMSE vector combining as a function of the number of antennas $(M)$. In a system with 4, 9 and 16 cells, with $K=20$ and $\Gamma=30 \mathrm{~dB}$.

\section{B. Simulation}

To validate the proposed combined vector in larger multicell scenarios, this work used different simulations based on the running example and parameters presented in [4], [7], [8]. Those systems are composed by $L$ cells deployed in a square area of $1 \mathrm{~km}^{2}$ using a universal pilot, where $L$ is a square number. Each cell has a single Base Station (BS) which serves $K$ users. And every BS has $M$ antennas, that are deployed next to each other in a uniform linear array (ULA). This simulation uses one-ring channel model for each UE. ${ }^{5}$

\section{NUMERICAL RESULTS}

In this section we will first compute the SIR heuristic of each UE in our experiment and plot them according to each UE location. Second we evaluate the impact of the SIR threshold $(\Gamma)$ on the PM-MMSE performance based on

\footnotetext{
${ }^{5}$ For more details of this simulation and its parameters, we strongly suggest refering to [4], [7], [8].
}

the data collected in our experiment. Finally with simulation results we asset the performance of the proposed PM-MMSE combined vector in comparison with other MMSE schemes, over SE, area SE and computational cost, for multi-cell scenarios.

\section{A. Experiment results}

The SIR heuristic from (12) for each inter-cell UE $i$ is plotted in Fig. 2 and Fig. 3 according to its location. It is noticeable first, that not all the UE contributes with the same SIR, and its value in this specific scenario varies from $-5 \mathrm{~dB}$ to $20 \mathrm{~dB}$. Second, as it is expected most of the users which cause strong inter-cell interference or low SIR, giving rise to a negative SIR heuristic, are located next to the adjacent cell. Third, despite that the scenario was deployed symmetrically the inter-cell SIR is not, that due to environmental effects of this particular experiment. Notice that although we compare the power received at BS1 from the neighboring cell with the strongest power from BS1, and still the SIR can be as low as $-5 \mathrm{~dB}$, meaning that the adjacent has a received power in the neighbouring cell that is $5 \mathrm{~dB}$ higher.

The key parameter for PM-MMSE is the SIR threshold $\Gamma$. To show its impact on SE from our two-cell outdoor experiment, the result is presented in Fig. 4 for M-MMSE, PM-MMSE and S-MMSE combining vectors, under different $\Gamma$ values. As discussed in Section III-B, small $\Gamma$ values gives the performance closer to S-MMSE, and high values to $\mathrm{M}$ MMSE. The obtained results show that when $\Gamma=0 \mathrm{~dB}$ an average of $Q=4$ is obtained in PM-MMSE (from a total of 18 inter-cell channels) and its SE increases $25 \%$ from $\mathrm{S}$ MMSE. When $\Gamma=2 \mathrm{~dB} Q=9$, and SE approaches $80 \%$ of the SE of M-MMSE. From $\Gamma=5 \mathrm{~dB}(Q=12)$ and onwards we can see that the remaining inter-cell interference do not impact much to the SE.

\section{B. Simulation results}

In the simulation for multi-cell scenarios, users are spread over an area of $1 \mathrm{~km}^{2}$. Users which are further to the analyzed cell will cause weak inter-cell interference, therefore, $\Gamma$ will have a higher value compared to the two-cell experiment.

Fig. 5 shows SE between PM-MMSE $(\Gamma=30 \mathrm{~dB})$ and M-MMSE for 4, 9 and 16 cells. We can see that when the number of cells increases (also density of users in the same area), the SE reduces because of larger channel estimation errors caused by pilot contamination and an increased of total intra and inter-cell interference. Moreover, when $L$ increases, PM-MMSE is closer to M-MMSE, since the size of the cells are smaller and more inter-cell UEs have a higher SIR below $\Gamma$.

In Table II, a numerical comparison for all MMSE combining vectors based on SE and area SE is presented. We can see that PM-MMSE achieves at least $94 \%$ of M-MMSE SE when $L=4$ and $96 \%$ when $L=9,16$. As was mentioned before, when the number of cells increase the SE per cell is reduced, but also systems with a large number of cells have a better area SE. It is worth to mention that for cases of $L=$ 
TABLE II

AREA SPECTRAL EFFICIENCY COMPARISON FOR MULTI-CELL SCENARIOS, WITH $M=100, K=20$ AND $\Gamma=30 \mathrm{~dB}$.

\begin{tabular}{|c|c|c|c|c|c|c|c|}
\hline \multirow{2}{*}{ Number of cells } & \multirow{2}{*}{ Area per cell $\left[\mathrm{km}^{2}\right]$} & \multicolumn{3}{|c|}{ SE [bits/s/Hz/cell] } & \multicolumn{3}{c|}{ Area SE [bits/s/Hz/km $\left.{ }^{2}\right]$} \\
\cline { 3 - 8 } & & M-MMSE & PM-MMSE & S-MMSE & M-MMSE & PM-MMSE & S-MMSE \\
\hline 4 & 0.2500 & 82.343 & 77.509 & 68.752 & 329.372 & 310.036 & 275.008 \\
\hline 9 & 0.0900 & 62.926 & 61.340 & 54.523 & 699.177 & 681.555 & 605.806 \\
\hline 16 & 0.0625 & 43.778 & 42.492 & 37.733 & 700.448 & 679.872 & 603.720 \\
\hline
\end{tabular}

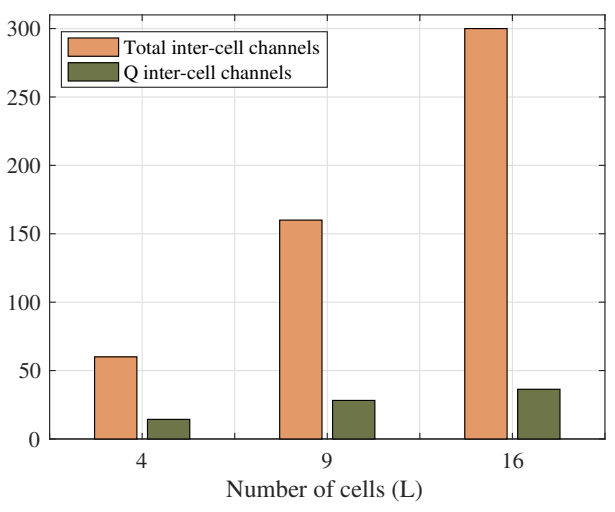

Fig. 6. Total number of inter-cell channels per cell to compute $\mathbf{V}_{\mathrm{M}-\mathrm{MMSE}}$ and the average total number of inter-cell channels $Q^{j}$ used to obtain $\mathbf{V}_{\text {PM-MMSE. }}$ For a simulation system with $M=100, K=20$ and $\Gamma=30 \mathrm{~dB}$.

9 and 16, the area SE for P-MMSE and M-MMSE are close as well.

Fig. 6, presents the average number of $Q$ inter-cell channels used by PM-MMSE versus the total number of inter-cell channels used by M-MMSE to obtain the SE in Fig. 5 when $M=100$. We can see that the number of inter-cell channels required by M-MMSE is linear dependent on the number of cells $(L-1) K$. On the contrary, $Q$ increases as a function of $K$ and SIR, and in the case of $L=16, Q$ is around a tenth of the total number of inter-cell channels.

Fig. 7 presents the total number of complex multiplications needed by each combining vector to achieve their SE in Fig. 5. Considering the number of complex multiplications from M-MMSE scheme, and the largest $Q$ value in the system, PM-MMSE reduces the computation up to $40 \%$ in a system with 16 cells.

\section{CONCLUSIONS}

This paper presents a compelling new vector combining method called PM-MMSE, which uses partial inter-cell information based on inter-cell SIR channel selection. This new scheme is capable of achieving high SE while reducing computational cost. It is worth nothing that in ultra dense scenarios with 16 cells and 320 users, PM-MMSE reaches $96 \%$ of the optimal spectral efficiency per cell, using on average a tent of the total inter-cell channels. As for the computation cost, in the same scenario, PM-MMSE requires only $60 \%$ of the total number of multiplications used by M-MMSE. We also propose a SIR parameter, which is used to control the trade-off between computational cost

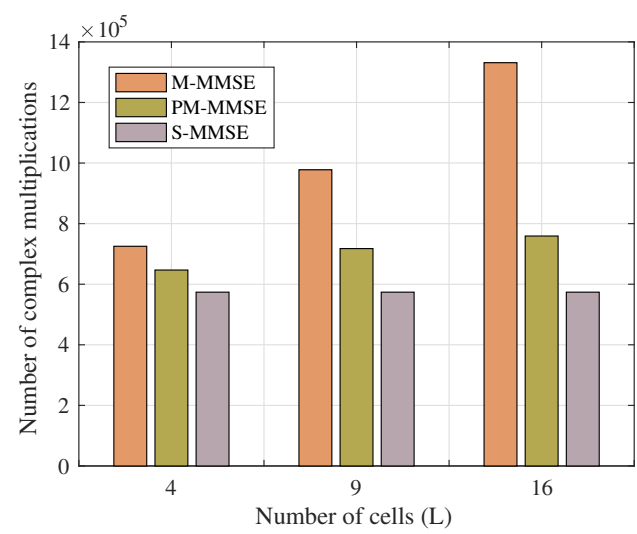

Fig. 7. Total number of complex multiplications per coherence block for M-MMSE, PM-MMSE (with the largest $Q$ ) and S-MMSE combining vector, in function of $L$. Considering $M=100, K=20$ and $\Gamma=30 \mathrm{~dB}$

and spectral efficiency. Multi-cell simulations along with an two-cell outdoor experiment for massive MIMO systems validate the efficiency of the proposed partial multi-cell MMSE combining vector.

\section{ACKNOWLEDGMENT}

This work was funded by the European Union's Horizon 2020 under grant agreement no. 732174 (ORCA project).

\section{REFERENCES}

[1] S. Malkowsky, J. Vieira, L. Liu, P. Harris, K. Nieman, N. Kundargi, I. C Wong, F. Tufvesson, V. Öwall, and O. Edfors, "The world's first realtime testbed for massive mimo: Design, implementation, and validation," IEEE Access, vol. 5, pp. 9073-9088, 2017.

[2] T. L. Marzetta, "Noncooperative cellular wireless with unlimited numbers of base station antennas," IEEE Transactions on Wireless Communications, vol. 9, pp. 3590-3600, Nov 2010.

[3] X. Li, E. Björnson, E. G. Larsson, S. Zhou, and J. Wang, "A multi-cell mmse detector for massive mimo systems and new large system analysis," in 2015 IEEE Global Communications Conference (GLOBECOM), pp. 1-6, Dec 2015.

[4] E. Björnson, J. Hoydis, and L. Sanguinetti, "Massive MIMO networks: Spectral, energy, and hardware efficiency," Foundations and Trends ${ }^{\circledR}$ in Signal Processing, vol. 11, no. 3-4, pp. 154-655, 2017.

[5] A. P. Guevara, C. Chen, and S. Pollin, "Hardware and spectrum sharing for distributed massive mimo," in 2018 52nd Asilomar Conference on Signals, Systems, and Computers, pp. 619-623, Oct 2018.

[6] "5g massive mimo testbed: From theory to reality." White Paper, August 2017.

[7] E. Björnson, E. G. Larsson, and M. Debbah, "Massive mimo for maximal spectral efficiency: How many users and pilots should be allocated?," IEEE Transactions on Wireless Communications, vol. 15, pp. 1293-1308, Feb 2016.

[8] X. Li, E. Björnson, E. G. Larsson, S. Zhou, and J. Wang, "Massive mimo with multi-cell mmse processing: exploiting all pilots for interference suppression," EURASIP Journal on Wireless Communications and Networking, vol. 2017, p. 117, Jun 2017. 